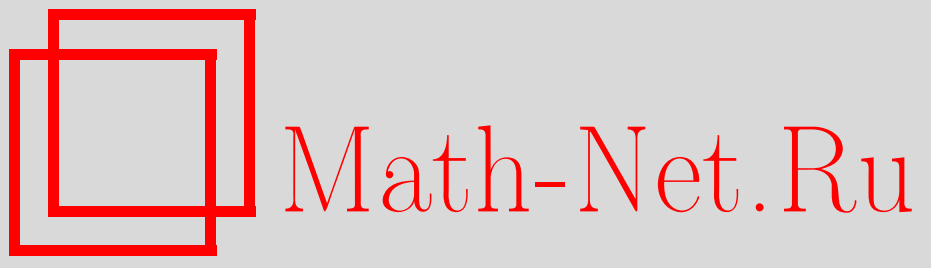

Обцероссийский математический портал

В. И. Малыхин, Новое о вполне ограниченных группах, $M a-$ тем. заметки, 1999, том 65, выпуск 3, 474-477

DOI: https://doi.org/10.4213/mzm1073 
Использование Общероссийского математического портала Math-Net.Ru подразумевает, что вы прочитали и согласны с пользовательским соглашением http://www. mathnet.ru/rus/agreement

Параметры загрузки:

IP: 44.207 .124 .84

26 апреля 2023 г., 03:02:09

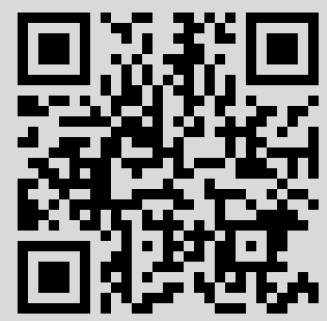




\section{НОВОЕ О ВПОЛНЕ ОГРАНИЧЕННЫХ ГРУППАХ}

\section{В.И. Малыхин}

Топологическая группа назьвается вполне ограниченной, если для любой окрестности единицы есть конечное число ее сдвигов (правых или левых, все равно), в сумме покрывающих всю группу. В классе хаусдорфовых топологий (далее все топологии предполагаются таковыми) вполне ограниченные группы - это в точности подгруппы компактных групп. На любой абелевой группе существует и единственна максимальная вполне ограниченная топология. В этой топологии нет сходящихся последовательностей.

В связи с этим было предпринято изучение более слабых феноменов сходимости. Например, Е. Ван Дауэн [1] задал вопрос о наличии незамкнутых дискретных подмножеств в бесконечных вполне ограниченных группах. Положительный ответ на этот вопрос дали М. И. Урсул [2] и затем И.В. Протасов [3]. Он доказал следующую теорему.

ТЕОрема. В бесконечной вполне ограниченной группе существует незамкнутое дискретное подмнохество.

Дискретность подмножества означает, что для всякой его точки существует окрестность, не содержащая других точек этого подмножества.

Из доказательства Протасова легко усматривается следующая немного более сильная

ТЕОрема 1. В бесконечной вполне ограниченной группе существует незамкнутое сильно дискретное подмножество.

(C) В. И. МАлыхин

1999 
Сильная дискретность подмножества означает, что у точек этого подмножества существует дизъюнктная система окрестностей.

Таким образом, хотя сходящихся последовательностей в бесконечной вполне ограниченной группе может не быть, к каждой точке такой группы накапливается некоторое сильно дискретное подмножество. Однако оказывается, что некоторый слабый аналог сходящейся последовательности присутствует во всякой бесконечной вполне ограниченной группе.

ОПРЕДЕЛЕНИЕ 1. Счетное бесконечное дизъюнктное семейство конечных подмножеств называется слабой последовательностью, сходящейся $\kappa$ точке, если каждая окрестность этой точки пересекает почти все члены семейства (за исключением конечного их числа).

Обычная сходящаяся к точке последовательность представляет собой счетное бесконечное множество точек такое, что каждая окрестность этой точки содержит почти всю последовательность (за исключением конечного числа ее членов).

Видно, что сходящаяся слабая последовательность похожа на обычную - получается из нее как бы "раздуванием" ее точек до конечных подмножеств. В частности, если $\mathscr{A}$ - сходящаяся слабая последовательность к точке $x$, то всякое бесконечное подсемейство $\mathscr{B} \subset \mathscr{A}$ также есть сходящаяся к той же точке слабая последовательность.

ТЕОРема 2. В бесконечной группе для каждой точки существует слабая последовательность, сходящаяся в любой вполне ограниченной групповой топологии к этой точке.

Перед тем, как доказывать эту “топологическую" теорему, проанализируем абстрактные счетные бесконечные группы.

ОПРЕДЕЛЕНИЕ 2 . Подмножество $X$ группы $G$ называется большим, если найдется конечное подмножество $K \subset G$ такое, что $X \cdot K=G$ или $K \cdot X=G$.

Отметим, что если $X$ - большое подмножество, то всякое его содержащее есть тоже большое подмножество. Семейство больших подмножеств обозначим через Big.

Пусть $X$ - бесконечное множество. Рассмотрим множество двоичных функций, определенных на $X$, как топологическое пространство $2^{X}$ с топологией декартова произведения несвязных двоеточий $2=\{0,1\}$. Следовательно, $2^{X}$ есть не что иное, как канторов куб. Подмножеству $A \subset X$ сопоставим его характеристическую функцию $\chi_{A} \in 2^{X}$. В $2^{X}$ рассмотрим счетно аддитивную меру $\mu$ как на произведении $|X|$ числа двоеточий с мерой каждой точки $1 / 2$. Ясно также, что $2^{X}$ есть компактная топологическая булева группа. В частности, сдвиг на 1 (функция, тождественно равная 1) не меняет категорию и меру множества.

Лемма. В счетной бесконечной әруппе $G$ :

а) множество $\mathscr{D}(\mathrm{Big})=\left\{\chi_{A}: A \in \mathrm{Big}\right\}$ есть подмножество первой категории и нуль-мнохество в $2^{G}$

б) существует счетное бесконечное дизвюнктное семейство конечных подмножсеств $\mathscr{F}$ такое, что каждое большое подмножество группы пересекает почти все члены семейства $\mathscr{F}$ (за исключением конечного их числа).

Неформально утверждение а) можно сфформулировать следующим образом: семейство больших подмножеств группы $G$ есть множество первой категории и нуль-множество в $2^{G}$.

ДокАЗАТЕЛЬСтво ЛЕммы. а) Так как семейство всех конечных подмножеств счетного множества счетно, достаточно доказать, что для каждого конкретного конечного подмножества $K \subset G$ множество $\mathscr{D}(K)=\left\{\chi_{X}: X \cdot K=G\right.$ или $\left.K \cdot X=G\right\}$ нигде не плотно в $2^{G}$.

Произвольное непустое открыто-замкнутое подмножество в $2^{G}$ задается парой дизъюнктных конечных множеств $A, B \subset G$ и есть $V(A, B)=\left\{\chi_{X}: A \subset X, B \cap X=\varnothing\right\}$. Пусть $p$ - произвольная точка из $G \backslash(A K \cup K A)$. Так как $A$ конечно, то $\left(p K^{-1} \cup K^{-1} p\right) \cap A=\varnothing$. Пусть $B^{\prime}=B \cup p K^{-1} \cup K^{-1} p$. Тогда $B^{\prime} \cap A=\varnothing$. Ясно, что $V\left(A, B^{\prime}\right) \subseteq V(A, B)$. Если $\chi_{X} \in V\left(A, B^{\prime}\right)$, то $X \cap B^{\prime}=\varnothing$, а значит, $\left(p K^{-1} \cup K^{-1} p\right) \cap X=\varnothing$. Следовательно, $p \notin X K \cup K X$ и потому $X K \cup K X \neq G$. Тем самым, $V\left(A, B^{\prime}\right) \cap \mathscr{D}(K)=\varnothing$. 
Доказано, что $\mathscr{D}(K)$ нигде не плотно и, следовательно, первая часть утверждения а) леммы также установлена.

Докажем, что $\mathscr{D}(K)$ - нуль-множество. Пусть $\mathscr{E}=\left\{(A, B): A, B \subset G,|A|,|B|<\aleph_{0}, A \cap B=\varnothing\right\}$. Из проведенного выше доказательства первой части утверждения а) леммы видно, что в каждом $V(A, B)$ для $(A, B) \in \mathscr{E}$ найдется $V\left(A, B^{\prime}\right)$ дизъюнктное с $\mathscr{D}(K)$, причем $\left|B^{\prime}\right| \leqslant|B|+2|A| \cdot|K|$. Пусть $E$ есть объединение всех таких $V\left(A, B^{\prime}\right)$. Тогда $E \cap \mathscr{D}(K)=\varnothing$. Ясно, что $E$ измеримо. Докажем, что $S=2^{G} \backslash E$ имеет меру 0. Предположим противное. Тогда по теореме Лебега (см. [4, c. 35$])$ в $S$ найдется точка $x$ плотности 1 . Это означает, что для любого $\varepsilon>0$ существует окрестность $V(A, B)$ точки $x$ такая, что $\mu(V(A, B) \cap S) \geqslant(1-\varepsilon) \mu(V(A, B))$. Но для $\varepsilon<2^{-2|A| \cdot|K|}$ нельзя найти такой окрестности. Доказательство утверждения а) леммы завершено.

б) Заметим, что семейство Big обладает следуюшим свойством:

*) если $X \in \operatorname{Big}$ и $Y \supseteq X$, то $Y \in$ Big.

М. Талагран доказал, что если семейство $\mathscr{B}$ подмножеств множества $G$ обладает свойством $*$ ) и $\mathscr{D}(\mathscr{B})=\left\{\chi_{B}: B \in \mathscr{B}\right\}$ есть множество первой категории в $2^{G}$, то существует счетное бесконечное дизъюнктное семейство $\mathscr{F}$ конечных подмножеств $G$ такое, что каждый элемент семейства $\mathscr{B}$ пересекает почти все члены семейства $\mathscr{F}$ (за исключением конечного их числа, см. [5, доказательство теоремы 21]). Доказательство леммы завершено.

Заметим, что во вполне ограниченной группе всякое подмножество с непустой внутренностью есть большое подмножество (см. в начале заметки характеристику вполне ограниченных групп). Поэтому из утверждения б) леммы следует

Теорема 3. В счетной бесконечной вполне ограниченной группе существует счетное бесконечное дизгюнктное семейство конечных подмножеств F्F такое, что для любой вполне ограниченной групповой топологии каждое непустое открытое в ней подмножество пересекает почти все члены $\mathscr{F}$. Следовательно, $\mathscr{F}$ есть слабая последовательность, сходящаяся в этой топологии к любой точке группы.

ДОКАЗАТЕЛЬСТВо ТЕОРЕМЫ 2. Заметим, что подгруппа вполне ограниченной группы сама вполне ограниченная. Пусть $G$ - какая-нибудь счетная бесконечная подгруппа рассматриваемой группы. Тогда из теоремы 3 следует наличие слабой последовательности в подгруппе $G$, сходящейся к каждой точке этой подгруппы в любой вполне ограниченной групповой топологии.

Теорема 4. В счетной бесконечной вполне ограниченной группе $G$ :

а) семейство подмножеств с непустой внутренностью есть множество первой категории и нуль-множество в $2^{G}$;

б) семейство подмножеств, не являюшихся всюду плотными, также есть множество первой категории и нуль-множество;

в) семейство всюду плотных подмножеств есть дополнение ко мнохеству первой категории и имеет меру 1 ;

г) семейство множеств, которые и сами и дополнения к которым всюду плотны, есть дополнение $к$ множеству первой категории и имеет меру 1.

ДокАЗАтельство. Разумеется, если говорить совершенно точно, то речь идет о характеристических функциях элементов указанных семейств. Ясно, что элементы семейства б) сдвигом на 1 в группе $2^{G}$ переходят в элементы семейства а). Ясно также, что семейство в) есть просто дополнение в $2^{G}$ семейства б). Далее, обозначим семейство в) через $\mathscr{W}$. Тогда очевидно, что $\mathscr{W} \cap(1-\mathscr{W})$ есть множество меры 1 и дополнительное к множеству первой категории.

Отметим, что, как следует из утверждения б), дискретных подмножеств, тем более дискретных незамкнутых, "мало", так что теорема Протасова и теорема 1 не могли быть получены из категорных соображений.

В [6] введено понятие сверхразложимости пространства. Для счетного пространства это означает существование несчетного семейства плотных подмножеств, а пересечение любых двух различных членов этого семейства нигде не плотно. Несложно доказать, что сверхразложимое пространство имеет бесконечное дизъюнктное семейство плотных подмножеств, т.е. такое пространство бесконечно разложимо. В [7] доказана бесконечная разложимость вполне ограниченной группы. 
ТЕОРема 5. В счетной бесконечной вполне ограниченной әруппе существует почти дизгюнктное семейство мощности $2^{\aleph_{0}}$ подмнохеств, плотных в любой вполне ограниченной групповой топологии. Таким образом, это семейство обеспечивает сверхразложимость любой вполне ограниченной групповой топологии на этой әруппе.

ДоКАЗАТЕЛЬство. Пусть $\mathscr{F}$ - семейство из теоремы 4 . Пусть $\Sigma$ - почти дизъюнктное семейство мощности $2^{\aleph_{0}}$ бесконечных подмножеств $\mathscr{F}$. Тогда $\{\bigcup \sigma: \sigma \in \Sigma\}$ - искомое семейство.

\section{СПИСОК ЦИТИРОВАННОЙ ЛИТЕРАТУРЫ}

1. van Dauwen E. K. // Topology Appl. 1990. V. 34. Р. 69-91. 2. Урсул М. И. // Изв. АН Республики Молдова. 1991. №1. С. 67-69. 3. Протасов И. В. // Матем. заметки. 1994. Т. 55. №1. С. 150-151. 4. Окстоби Дж. Мера и категория. М.: Мир, 1974. 5. Telagrand M. // Studia Math. 1980. V. 67. P. 13-43. 6. Malykhin V. I. Irresolvability is not descriptively good // Comment. Math. Univ. Carolin. (to appear). 7. Malykhin V. I., Protasov I. V. // Topology Appl. 1996. V. 20. P. 1-6.

Государственная академия управления им. С. Орджоникидзе 